\title{
Evolution of incidence of audiovestibular disorders during the pandemic COVID-19 period
}

\author{
Chun-Hao Chao ${ }^{1} \cdot$ Yi-Ho Young $^{1}$ (I)
}

Received: 10 July 2021 / Accepted: 9 August 2021 / Published online: 13 August 2021

(c) The Author(s), under exclusive licence to Springer-Verlag GmbH Germany, part of Springer Nature 2021

\begin{abstract}
Purpose Despite sporadic case reports describing hearing problems in patients with coronavirus disease 2019 (COVID-19), whether COVID-19 affects the audiovestibular system remains unclear. This study assessed the evolution of incidence of audiovestibular disorders during the pandemic COVID-19 period.

Method Three audiovestibular disorders namely, sudden sensorineural hearing loss (SSHL), autonomic dysfunction, and Meniere's disease (MD) were analyzed and compared from 2016 to 2020.

Results The annual new cases at our clinic comprised overall 2107, 1997, 1984, 2068, and 1829 from 2016 to 2020, respectively, and the respectively annual cases of SSHL were 54, 46, 42, 45 and 38. Accordingly, annual incidences of SSHL in relation to overall cases of audiovestibular disorders were $2.6 \%, 2.3 \%, 2.1 \%, 2.2 \%$ and $2.1 \%$ from 2016 to 2020 , respectively, exhibiting a non-significant difference $(p>0.05)$. In contrast, incidence of autonomic dysfunction in the year 2020 was $15.3 \%$, which revealed significantly higher than 8.5-13.1\% from 2016 to $2019(p<0.001)$. Restated, the incidence of autonomic dysfunction in 2020 displayed a significantly higher percentage than the other 4 years. Conversely, the incidence of MD in 2020 was $9.8 \%$, showing a significant decline compared with the other 4 years $(12.6-15.6 \%$ from 2016 to $2019, p<0.001)$, Conclusion Evolution of incidence of audiovestibular disorders during the pandemic COVID-19 period revealed increase in the incidence of autonomic dysfunction and decrease in that of $\mathrm{MD}$, while incidence of SSHL remained unchanged from 2016 to 2020. Thus, the SARS-CoV-2 may less affect the audiovestibular system.
\end{abstract}

Keywords Autonomic dysfunction · Coronavirus disease 2019 (COVID-19) · Meniere's disease · Sudden sensorineural hearing loss

\section{Introduction}

In December 2019, sporadic cases of atypical pneumonia were consecutively encountered in Wuhan, China, and a novel virus termed severe acute respiratory syndrome coronavirus 2 (SARS-CoV-2) was later identified as the etiology. The World Health Organization (WHO) declared the global outbreak of coronavirus and coined the term "Coronavirus Disease 2019 (COVID-19)". By the end of 2020, based on WHO COVID-19 dashboard (2020), more than 90 million patients with COVID-19 had been confirmed worldwide, with a mortality rate of $2.2 \%$ [1]. Typical symptoms of COVID-19 comprised fever, cough, fatigue, shortness of

Yi-Ho Young

youngyh@ntu.edu.tw

1 Department of Otolaryngology, National Taiwan University Hospital, 1 Chang-te St., Taipei, Taiwan breath, etc. [2]. In addition, loss of smell and taste were associated neurological symptoms ranging from 5-98\% and 6-90\% in prevalence, respectively, likely due to direct invasion of the SARS-CoV-2, or inflammatory response by cytokine storm [3-5]. Further, inner ear symptoms, i.e., tinnitus, hearing loss, and balance disorders were also mentioned in sporadic case reports of COVID-19 [6-9]. Whether COVID-19 affects the audiovestibular system remains unclear. If hearing impairment relates to COVID-19, then the incidence of sudden sensorineural hearing loss (SSHL), like hyposmia and hypogeusia, may have increased during the pandemic period of COVID-19. Hence, this study compared evolution of incidence of three audiovestibular disorders namely, SSHL, autonomic dysfunction, and Meniere's disease (MD) from 2016 to 2020. 


\section{Methods}

From January 2016 to December 2020, patients with inner ear symptoms and pathological eye movements who visited neurotological clinic of the university hospital for the first time were enrolled in this study. All patients underwent an inner ear test battery comprising audiometry, ocular vestibular-evoked myogenic potential (oVEMP) test, cervical VEMP (cVEMP) test, and bithermal caloric test.

Diagnosis of SSHL is defined as a rapid decline ( $<3$ days) of more than $30 \mathrm{~dB}$ of sensorineural hearing loss in at least three contiguous frequencies without an identifiable cause [10]. Diagnosis of MD was based on the guidelines proposed by the American Academy of Otolaryngology-Head and Neck Surgery and new diagnostic criteria defined by the Barany Society [11, 12]. Diagnosis of autonomic dysfunction was based on the literature [13], namely, (1) non-vestibular dizziness, e.g., light headedness; (2) provocation by sudden postural change or orthostatic hypotension by Schellong test; (3) duration of seconds to several minutes; and (4) at least five of following the symptoms: anxiety, chronic fatigue, cold extremities, fainting, frequent headache, gastrointestinal symptoms, medication intolerance, palpitation, or sleeping disorder, but no rotational vertigo.

This study was approved by the institutional review board of the university hospital, and each subject signed the informed consent to participate.

\section{Statistical methods}

The annual incidence of each disorder from 2016 to 2020 was compared by $2 \times 5$ Chi-square test. Associations between each year and cases of audiovestibular disorder were determined by binary logistic regression. The produced coefficients and standard errors were exponentiated to create odds ratio (OR) with their respective $95 \%$ confidence interval (CI). A significant difference indicates $p<0.05$.

\section{Results}

\section{Evolution of incidence of SSHL}

From January 2016 to December 2020, the annual new cases of audiovestibular disorders at the neurotological clinic of a university hospital were 2107, 1997, 1984, 2068, and 1829, respectively, and the respectively annual cases of SSHL were 54, 46, 42, 45 and 38 (Table 1). Accordingly, annual incidences of SSHL in relation to overall cases of audiovestibular disorders were $2.6 \%, 2.3 \%, 2.1 \%, 2.2 \%$ and $2.1 \%$ from 2016 to 2020, respectively, exhibiting a non-significant difference ( $p>0.05$, Fig. 1). Additionally, binary logistic regression (SSHL vs. non-SSHL cases) to compare 2020 (COVID year) with the other 4 years (2016-2019) yielded no significant difference in the odds ratio $(p>0.05$, Table 1$)$.

\section{Evolution of incidence of autonomic dysfunction}

Table 2 summarizes the incidence of autonomic dysfunction from 2016 to 2020. Unlike SSHL, the incidence of autonomic dysfunction in the year 2020 was $15.3 \%$, which revealed significantly higher than $8.5-13.1 \%$ from 2016 to 2019 ( $p<0.001$, Fig. 2). Restated, the incidence of

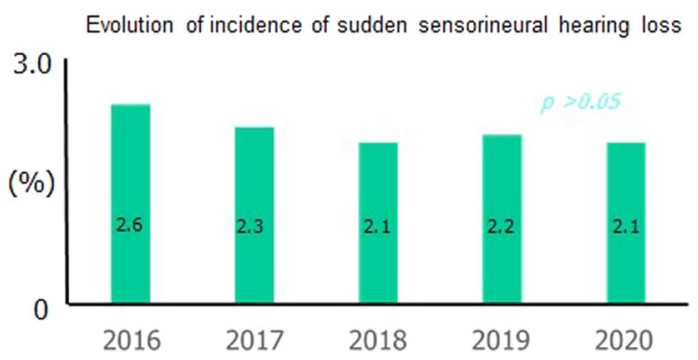

Fig. 1 The annual incidences of sudden sensorineural hearing loss in relation to overall cases of audiovestibular disorders are $2.6 \%, 2.3 \%$, $2.1 \%, 2.2 \%$ and $2.1 \%$ from 2016 to 2020 , respectively, exhibiting a non-significant difference $(p>0.05)$
Table 1 Incidence of sudden sensorineural hearing loss (SSHL) in relation to overall neurotological cases

\begin{tabular}{lllccc}
\hline Year & $\begin{array}{l}\text { SSHL } \\
\text { Case no }\end{array}$ & $\begin{array}{l}\text { Overall } \\
\text { Case no }\end{array}$ & Incidence & Odds ratio (95\% CI) & $p$ value $^{\#}$ \\
\hline 2016 & 54 & 2107 & $2.6 \%$ & $1.240(0.815-1.886)$ & 0.316 \\
2017 & 46 & 1997 & $2.3 \%$ & $1.111(0.720-1.716)$ & 0.634 \\
2018 & 42 & 1984 & $2.1 \%$ & $1.019(0.654-1.588)$ & 0.933 \\
2019 & 45 & 2068 & $2.2 \%$ & $1.048(0.678-1.622)$ & 0.832 \\
2020 (COVID-19) & 38 & 1829 & $2.1 \%$ & 1 & \\
$p$ value* & & & $>0.05$ & & \\
\hline
\end{tabular}

CI confidence interval

$* 2 \times 5$ Chi-square test

\#Binary logistic regression, when compared with year 2020 
Table 2 Incidence of autonomic dysfunction in relation to overall neurotological cases

\begin{tabular}{lllrlr}
\hline Year & $\begin{array}{l}\text { Autonomic } \\
\text { dysfunction } \\
\text { Case no }\end{array}$ & $\begin{array}{l}\text { Overall } \\
\text { Case no }\end{array}$ & Incidence & Odds ratio (95\% CI) & $p$ value $^{\#}$ \\
\hline 2016 & 180 & 2107 & $8.5 \%$ & $0.515(0.422-0.629)$ & $<0.001$ \\
2017 & 197 & 1997 & $9.9 \%$ & $0.606(0.498-0.735)$ & $<0.001$ \\
2018 & 258 & 1984 & $13.0 \%$ & $0.827(0.689-0.992)$ & 0.041 \\
2019 & 271 & 2068 & $13.1 \%$ & $0.838(0.697-0.999)$ & 0.048 \\
2020 (COVID-19) & 280 & 1829 & $15.3 \%$ & 1 & \\
$p$ value* & & & $<0.001$ & & \\
\hline
\end{tabular}

CI confidence interval

$* 2 \times 5$ Chi-square test

\#Binary logistic regression, when compared with year 2020

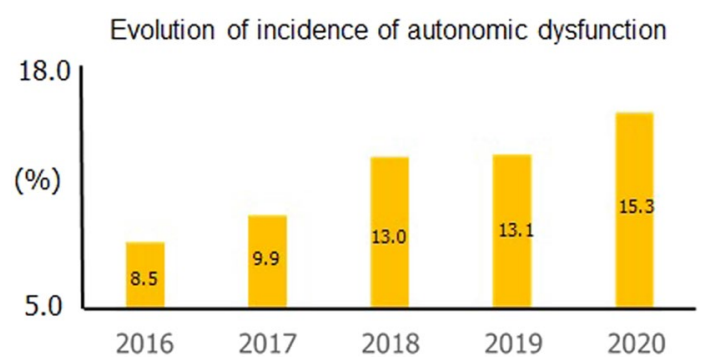

Fig. 2 The annual incidence of autonomic dysfunction in 2020 displays a significantly higher percentage than the other 4 years (2016$2019, p<0.001$ )

autonomic dysfunction in 2020 displayed a significantly higher percentage than the other 4 years. Additionally, binary logistic regression to compare 2020 with the other 4 years also yielded a significant difference in the odds ratio $(p<0.05$, Table 2).

\section{Evolution of incidence of MD}

Conversely, the incidence of MD in 2020 was $9.8 \%$, compared with $15.6 \%$ (2016), 14.6\% (2017), 12.3\% (2018) and $12.6 \%$ (2019), exhibiting a significant decline from 2016 to 2020 ( $p<0.001$, Fig. 3). Further, binary logistic regression also identified a significant difference in the odds ratio between 2020 and the other 4 years $(p<0.05$, Table 3$)$.

\section{Discussion}

In addition to respiratory symptoms, associated neurosensory deficits such as hyposmia and hypogeusia are frequently present in COVID-19 patients, likely due to direct invasions of the SARS-CoV-2 or inflammatory response by cytokine storm [14]. Meanwhile, tinnitus or hearing loss is occasionally mentioned in sporadic case reports

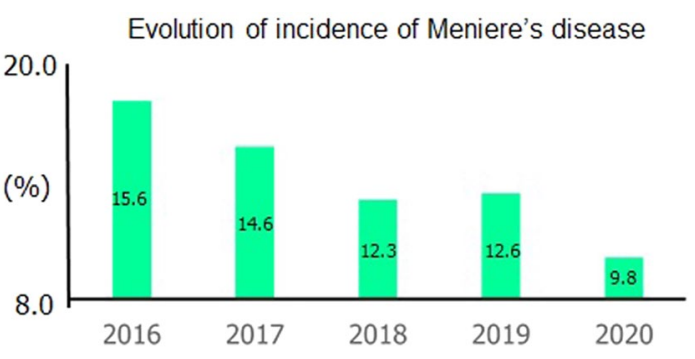

Fig. 3 The annual incidence of Meniere's disease in 2020 displays a significantly lower percentage than the other 4 years (2016-2019, $p<0.001)$

[6-9]. Comparing the annual incidence of SSHL at our hospital in 2020 with that in 2016-2019 (Fig. 1), no significant difference was identified over the past 5 years (Table 1), indicating that SSHL in COVID-19 patients is coincidental rather than a cause-and-effect relationship. Notably, some COVID-19 patients treated by chloroquine may experience hearing loss/tinnitus, and ototoxicity by chloroquine should be differentiated from SSHL [15]. Furthermore, those with slight hearing decline or a gradual or delayed loss, but not SSHL, otoscopy is essential to exclude concomitant ear infection.

During the pandemic period of COVID-19, more and more patients with autonomic dysfunction were experienced at our clinic (Fig. 2). Unlike SSHL, the incidence of autonomic dysfunction in $2020(15.3 \%)$ is significantly higher than the other 4 years (8.5-13.1\%, Table 2), probably because of increased psychological stress, panic, anxiety, or depression associated with social isolation in time of a pandemic COVID-19 period [16].

On the other hand, those who spent too much time focusing on the outbreak or the epidemic progression may also have developed autonomic dysfunction. As a result, it is not surprising that an increased numbers of patients with autonomic dysfunction visited our clinic in the most arduous year, 2020 (Fig. 2). 
Table 3 Incidence of Meniere's disease (MD) in relation to overall neurotological cases

\begin{tabular}{lllcrr}
\hline Year & $\begin{array}{l}\text { MD } \\
\text { Case no }\end{array}$ & $\begin{array}{l}\text { Overall } \\
\text { Case no }\end{array}$ & Incidence & Odds ratio (95\% CI) & $p$ value $^{\#}$ \\
\hline 2016 & 329 & 2107 & $15.6 \%$ & $1.641(1.358-1.982)$ & $<0.001$ \\
2017 & 291 & 1997 & $14.6 \%$ & $1.567(1.242-1.829)$ & $<0.001$ \\
2018 & 245 & 1984 & $12.3 \%$ & $1.246(1.021-1.521)$ & 0.031 \\
2019 & 261 & 2068 & $12.6 \%$ & $1.297(1.066-1.578)$ & 0.009 \\
2020 (COVID-19) & 180 & 1829 & $9.8 \%$ & 1 & \\
$p$ value* & & & $<0.001$ & & \\
\hline
\end{tabular}

$C I$ confidence interval

$* 2 \times 5$ Chi-square test

${ }^{\#}$ Binary logistic regression, when compared with year 2020
Conversely, the incidence of MD in 2020 (9.8\%) is significantly lower than the other 4 years $(12.6-15.6 \%$, Fig. 3 , Table 3) despite the pandemic environment. Although one may argue that MD patients did exist, yet they just never presented for medical care. Nevertheless, such reason opposed to the phenomenon that patient numbers of autonomic dysfunction increase.

In February 2020, the policy for eliminating the spread of COVID-19 was carried out by the government, based on the past experience on SARS in 2003 [17]. For example, enforcement of a 14-day quarantine period for travelers arriving from abroad is required. Airport checkpoints have already been set up to screen passengers, where they are given temperature checks, a nasopharyngeal swab for detecting the viral load, and asked to fill out health declaration forms. All subjects are required to wear masks in public areas. Those with positive cases of COVID-19 should wear N-95 masks and immediately be placed in isolation facilities with negative pressure. Meanwhile, all subjects are required to wear masks in public areas, i.e., hospital, school, theater, concert, market, gym, transportation, etc.

Many events are suggested to trigger Meniere attacks such as weather change, common cold, mental stress, etc. Mizukoshi et al. [18] reported that the Meniere attack is influenced by the passing of a cold front, defined as the following conditions, namely (1) fall in temperature by $>5^{\circ} \mathrm{C}$, (2) increased humidity, (3) strong wind $>10 \mathrm{~m} / \mathrm{s}$, (4) rain or snow, (5) thunder, and (6) wind shifting from south to north-west in Toyama Prefecture, Japan. However, because a cold front containing multiples factors, clinicians are not clear with which meteorological parameter is responsible for Meniere attack.

Wearing masks helps avoid significant temperature change and infection because the common cold is a precipitating factor for a Meniere attack [19, 20]. The advantage of wearing masks under weather change, pending further validation, may help eliminate the precipitating factors, i.e., common cold or temperature change for vertiginous attack. Hence, in the future, when facing seasonal change, i.e., a cold front, MD patients are advised to wear masks avoiding vertiginous attack.

\section{Conclusion}

Evolution of incidence of audiovestibular disorders during the pandemic COVID-19 period revealed increase in the incidence of autonomic dysfunction and decrease in that of MD, while incidence of SSHL remained unchanged from 2016 to 2020 . Thus, the SARS-CoV-2 may less affect the audiovestibular system.

Acknowledgements This study was supported by the National Science Council, Taiwan (Grant no. Most 109-2314-B-002-265).

\section{Declarations}

Conflict of interest The authors declare that they have no conflict of interest.

Sponsorships None.

\section{References}

1. WHO Coronavirus Disease (COVID-19) Dashboard (2020). https://covid19.who.int/. Accessed 11 Jan 2021

2. Mao L, Jin H, Wang M, Hu Y, Chen S, He Q, Chang J, Hong C, Zhou Y, Wang D, Miao X, Li Y, Hu B (2020) Neurologic manifestations of hospitalized patients with coronavirus disease 2019 in Wuhan, China. JAMA Neurol 77:683-690

3. Ellul MA, Benjamin L, Singh B, Lant S, Michael BD, Easton A, Kneen R, Defres S, Sejvar J, Solomon T (2020) Neurological associations of COVID-19. Lancet Neurol 19:767-783

4. Matschke J, Lütgehetmann M, Hagel C, Sperhake JP, Schröder AS, Edler C, Mushumba H, Fitzek A, Allweiss L, Dandri M, Dottermusch M, Heinemann A, Pfefferle S, Schwabenland M, Sumner Magruder D, Bonn S, Prinz M, Gerloff C, Püschel K, Krasemann S, Aepfelbacher M, Glatzel M, Matschke J (2020) Neuropathology of patients with COVID-19 in Germany: a postmortem case series. Lancet Neurol 19:919-929 
5. Liang Y, Xu J, Chu M, Mai J, Lai N, Tang W, Yang T, Zhang S, Guan C, Zhong F, Yang L, Liao G (2020) Neurosensory dysfunction: a diagnostic marker of early COVID-19. Int J Infect Dis 98:347-352

6. Almufarrij I, Uus K, Munro KJ (2020) Does coronavirus affect the audio-vestibular system? A rapid systematic review. Int J Audiol 59:487-491

7. Sriwijitalai W, Wiwanitkit V (2020) Hearing loss and COVID-19: a note. Am J Otolaryngol 41:102473

8. Koumpa FS, Forde CT, Manjaly JG (2020) Sudden irreversible hearing loss post COVID-19. BMJ Case Rep 13:e238419

9. Viola P, Ralli M, Pisani D, Malanga D, Sculco D, Messina L, Laria C, Aragona T, Leopardi G, Ursini F, Scarpa A, Topazio D, Cama A, Vespertini V, Quintieri F, Cosco L, Cunsolo EM, Chiarella G (2020) Tinnitus and equilibrium disorders in COVID-19 patients: preliminary results. Eur Arch Otorhinolaryngol. https:// doi.org/10.1007/s00405-020-06440-

10. Stachler RJ, Chandrasekhar SS, Archer SM et al (2012) Clinical practice guideline: sudden hearing loss. Otolaryngol Head Neck Surg 146(suppl 3):S1-35

11. Committee on Hearing and Equilibrium guideline for the diagnosis and evaluation of therapy in Meniere's disease (1995) Otolaryngol Head Neck Surg 113:181-185

12. Lopez-Escamez JA, Carey J, Chung WH, Goebel JA, Magnusson M, Mandalà M, Newman-Toker DE, Strupp M, Suzuki M, Trabalzini F, Bisdorff A (2015) Diagnostic criteria for Meniere's disease. J Vestib Res 25:1-7

13. Pappas DG Jr (2003) Autonomic dysfunction. Laryngoscope 113:1658-1671

14. Vallamkondu J, John A, Wani WY, Ramadevi SP, Jella KK, Reddy PH, Kandimalla R (2020) SARS-CoV-2 pathophysiology and assessment of coronaviruses in CNS diseases with a focus on therapeutic targets. Biochim Biophys Acta Mol Basis Dis 1866:165889

15. Karia RH, Nagraj S, Gupta I, Barua A, Kaur N, Singh H (2021) Hydroxychloroquine: a review of its safety and efficacy in COVID-19. J Fam Med Prim Care 10:1124-1133

16. Guessoum SB, Lachal J, Radjack R, Carretier E, Minassian S, Benoit L, Moro MR (2020) Adolescent psychiatric disorders during the COVID-19 pandemic and lockdown. Psychiatry Res 291:113264

17. Chen HC, Young YH, Luo JP, Tsai HJ, Ho SJ, Yeh CH, Huang LC (2003) Initial otolaryngological manifestations of severe acute respiratory syndrome in Taiwan. Arch Otolaryngol Head Neck Surg 129:1157-1160

18. Mizukoshi K, Watanabe Y, Shojaku H, Ito M, Ishikawa M, Aso S, Asai M, Motoshima H (1995) Influence of a cold front upon the onset of Meniére's disease in Toyama, Japan. Acta Otolaryngol Suppl 520:412-414

19. Schmidt W, Sarran C, Ronan N, Barrett G, Whinney DJ, Fleming LE, Osborne NJ, Tyrrell J (2017) The weather and Ménière's disease: a longitudinal analysis in the UK. Otol Neurotol 38:225-233

20. Gürkov R, Strobl R, Heinlin N, Krause E, Olzowy B, Koppe C, Grill E (2016) Atmospheric pressure and onset of episodes of Menière's disease-a repeated measures study. PLoS ONE 11:e0152714

Publisher's Note Springer Nature remains neutral with regard to jurisdictional claims in published maps and institutional affiliations. 